\title{
Turán densities of some hypergraphs related to $K_{k+1}^{k}{ }^{*}$
}

\author{
József Balogh${ }^{1}$, Tom Bohman², Béla Bollobás ${ }^{3}$, and Yi Zhao ${ }^{4}$ \\ ${ }^{1}$ Department of Mathematics, University of Illinois, 1409 W. Green Street Urbana, IL \\ 61801, jobal@math.uiuc.edu \\ ${ }^{2}$ Department of Mathematical Sciences, Carnegie Mellon University, Pittsburgh, PA 15213, \\ tbohman@math. cmu .edu \\ ${ }^{3}$ Trinity College, Cambridge CB2 1TQ England, B.Bollobas@dpmms.cam.ac.uk \\ ${ }^{3}$ Department of Mathematical Sciences, University of Memphis, Memphis, TN 38152, USA. \\ ${ }^{4}$ Department of Mathematics and Statistics, Georgia State University, Atlanta, GA 30303, \\ yzhao6@gsu.edu
}

February 1, 2012

\begin{abstract}
Let $B_{i}^{(k)}$ be the $k$-uniform hypergraph whose vertex set is of the form $S \cup T$, where $|S|=i$, $|T|=k-1$ and $S \cap T=\emptyset$, and whose edges are the $k$-subsets of $S \cup T$ that contain either $S$ or $T$. We derive upper and lower bounds for the Turán density of $B_{i}^{(k)}$ that are close to each other as $k \rightarrow \infty$. We also obtain asymptotically tight bounds for the Turán density of several other infinite families of hypergraphs. The constructions implying the lower bounds are derived from elementary number theory by probabilistic arguments, and the upper bounds follow from some results of de Caen, Sidorenko, and Keevash.
\end{abstract}

\section{Introduction}

Given an integer $k \geq 2$ and a set $V$ of size $|V| \geq k$, we denote by $\left(\begin{array}{l}V \\ k\end{array}\right)$ the family of all $k$-element subsets (in short, $k$-subsets) of $V$. A $k$-uniform hypergraph $H$ is an ordered pair $(V, E)$, where the edge set $E$ is a subfamily of $\left(\begin{array}{c}V \\ k\end{array}\right)$. Given two $k$-uniform hypergraphs $H$ and $G$, we call $G H$-free if $G$ does not contain $H$ as a subhypergraph. The extremal function $\operatorname{ex}(n, H)$ is defined as the maximum number of edges in an $H$-free $k$-uniform hypergraph on $n$ vertices, while the Turán density of $H$ is

$$
\pi(H)=\lim _{n \rightarrow \infty} \frac{\operatorname{ex}(n, H)}{\left(\begin{array}{l}
n \\
k
\end{array}\right)} .
$$

${ }^{*}$ The first author was supported by NSF CAREER Grant DMS-0745185, UIUC Campus Research Board Grant 11067, and OTKA Grant K76099. The second author was supported by NSF grant DMS-1001638. The third author was supported by NSF grants CNS-0721983, CCF-0728928 and DMS-0906634, ARO grant W911NF-06-1-0076, and TAMOP-4.2.2/08/1/2008-0008 program of the Hungarian Development Agency. The fourth author was supported NSA grant H98230-10-1-0165. 
For $k=2$ the Turán density of $H$ is determined by its chromatic number, namely, $\pi(H)=1-$ $\frac{1}{\chi(H)-1}$.

In the case $k \geq 3$ much less is known. Let $K_{r}^{k}$ be the complete $k$-uniform hypergraph on $r$ vertices. A classical problem of Turán [13] is to determine $\pi\left(K_{r}^{k}\right)$ when $r>k \geq 3$. Erdös [3] offered $\$ 500$ for determining $\pi\left(K_{k}^{r}\right)$ for any particular $k>r \geq 3$ and $\$ 1000$ for solving the problem for all $k$ and $r$. There are several constructions which show that $\pi\left(K_{4}^{3}\right) \geq \frac{5}{9}$ (see, e.g., [7]), while the best upper bound is $\pi\left(K_{4}^{3}\right) \leq 0.561666$ obtained by Razborov [10] (see Falgas-Ravry and Vaughan [4] for more recent results obtained using flag algebras). For $\pi\left(K_{k+1}^{k}\right)$, the natural asymptotic question remains open: the best known bounds for $\pi\left(K_{k+1}^{k}\right)[12,8]$ imply that

$$
1-\Theta\left(\frac{\ln k}{k}\right) \leq \pi\left(K_{k+1}^{k}\right) \leq 1-\Theta\left(\frac{1}{k}\right)
$$

In this paper we consider the asymptotic Turán densities of some classes of hypergraphs related to $K_{k+1}^{k}$. Given $2 \leq i \leq k$, define a $k$-uniform hypergraph $B_{i}^{(k)}$ as follows. The vertex set of $B_{i}^{(k)}$ is of the form $S \cup T$, where $S \cap T=\emptyset,|S|=i$ and $|T|=k-1$, and the edge set consists of all $k$-element subsets of $S \cup T$ containing either $S$ or $T$. Clearly, $B_{2}^{(k)}=K_{k+1}^{k}$. Concerning the other extreme value of $i$, Bohman, Frieze, Mubayi and Pikhurko [1] recently showed that as $k \rightarrow \infty$

$$
\pi\left(B_{k}^{(k)}\right)=1-(2+o(1))\left(\frac{\ln k}{k}\right) .
$$

As noted by Mubayi, the sequence of hypergraphs $B_{2}^{(k)}, B_{3}^{(k)}, \ldots, B_{k}^{(k)}$ starts with $K_{k+1}^{k}$ and ends with the graph $B_{k}^{(k)}$ studied in [1], this sequence gives us a natural progression from a classical hypergraph Turán problem to a problem whose asymptotic solution we know. Progress on the Turán densities for the hypergraphs $B_{i}^{(k)}$ with $2<i<k$ might shed some light on the limiting behavior of $\pi\left(K_{k+1}^{k}\right)$. In this paper we take the first tentative steps in this direction: we derive bounds resembling (1) for the hypergraphs $B_{k-i}^{(k)}$ and $B_{i}^{(k)}$ with $i$ fixed and $k$ tending to infinity.

Theorem 1. Fix $i \geq 1$. As $k \rightarrow \infty$ we have

$$
1-\Theta\left(\frac{\ln k}{k^{i}}\right) \leq \pi\left(B_{i+1}^{(k)}\right), \pi\left(B_{k-i}^{(k)}\right) \leq 1-\Theta\left(\frac{1}{k^{i}}\right) .
$$

Actually, Theorem 1 is the special case of the following more general statement.

Theorem 2. For every pair of integers $i, k$ satisfying $1 \leq i \leq \ln k /(10 \ln \ln k)$, we have

$$
1-(1+o(1)) \frac{12 i^{2} \ln k}{\left(\begin{array}{c}
k \\
i
\end{array}\right)} \leq \pi\left(B_{i+1}^{(k)}\right), \pi\left(B_{k-i}^{(k)}\right) \leq 1-\frac{1}{k-i+\left(\begin{array}{c}
k-1 \\
i
\end{array}\right)-1},
$$

where the o(1) term tends to 0 as $k \rightarrow \infty$, independently of $i$.

The upper bound in Theorem 2, which is actually valid for $i<k / 2$ as well, immediately follows from a result of Sidorenko [11], which relates the Turán density of a hypergraph to its size. The construction we used to prove the lower bound in Theorem 2 does not use the entire structure of the excluded hypergraph; it requires only the edges containing $S$ and a single edge containing $T$. 
Motivated by this observation, we define $\widehat{B}_{i}^{(k)}$ to be the $k$-uniform hypergraph whose vertex set is the disjoint union $S \cup T \cup\{v\}$, where $|S|=i$ and $|T|=k-1$, and whose edge set is

$$
\{T \cup\{v\}\} \cup\left\{S \cup T^{\prime}: T^{\prime} \in\left(\begin{array}{c}
T \\
k-i
\end{array}\right)\right\} .
$$

In other words, we obtain $\widehat{B}_{i}^{(k)}$ from $B_{i}^{(k)}$ by removing all of the edges that contain $T$ and adding a single new edge consisting of $T$ and a new vertex $v$. Applying to this hypergraph a result of de Caen [2] on the number of complete subhypergraphs in dense hypergraphs, we shall obtain asymptotically matching bounds for $\pi\left(\widehat{B}_{k-i}^{(k)}\right)$.

Theorem 3. Fix $i \geq 1$. We have $\pi\left(\widehat{B}_{k-i}^{(k)}\right)=1-\Theta\left(\frac{\ln k}{k^{i}}\right)$ as $k \rightarrow \infty$.

Finally, define a $k$-uniform hypergraph $C^{(k)}$ with $2 k-2$ vertices and $k$ edges as follows. The vertex set of $C^{(k)}$ has a partition into two $(k-1)$-sets: $V\left(C^{(k)}\right)=S \cup T$, with $S \cap T=\emptyset$ and $|S|=|T|=k-1$, and the edge set of $C^{(k)}$ consists of all $k$-sets containing $S$ and a single $k$-set containing $T$. By applying a result of Keevash [5] that relates the Turán density of a hypergraph to the maximal size of a forest contained in it, we shall obtain asymptotically matching bounds for $\pi\left(C^{(k)}\right)$.

Theorem 4. For $k \rightarrow \infty$, we have $\pi\left(C^{(k)}\right)=1-\Theta\left(\frac{\ln k}{k}\right)$.

While our upper and lower bounds for the Turán density of $B_{i}^{(k)}$ are close to each other, it would be nice to know which one is closer to the truth. We suspect that it should be closer to the upper bound. It would also be interesting to find a good lower bound on the Turán density of $B_{i}^{(k)}$ for $i$ near $k / 2$.

In Section 2 we prove the lower bounds in Theorems 2, 3, and 4, and in Section 3 the upper bounds.

\section{Lower Bounds}

Kim and Roush [6] constructed a dense $K_{k+1}^{k}$-free $k$-uniform hypergraph $\mathcal{H}_{k}$; let us start by recalling this construction. Let $\ell \approx \frac{k}{2 \ln k}$ be a prime number, and take an equipartition of the vertex set $[n]$ into $\ell$ sets $V_{1}, \ldots, V_{\ell}$. It is easy to see that almost all $k$-subsets of $[n]$ intersect each of the sets $V_{i}$. Define the weight of a subset $X$ of $[n]$ as $w(X)=\sum_{j=1}^{\ell} j\left|X \cap V_{j}\right|(\bmod \ell)$. Let $i \in \mathbb{Z}_{\ell}$ be chosen such that the number of $k$-sets having weight $i$ is minimal. The edge set of $\mathcal{H}_{k}$ will consist of all $k$-sets that intersect all $V_{1}, \ldots, V_{\ell}$ except for those with weight $i$. In this section we shall generalize this construction.

Definition 5. Given positive integers $i, \ell$ and $k$, an $i$-fold sum cover of $\mathbb{Z}_{\ell}$ is a multi-set $X$ of elements of $\mathbb{Z}_{\ell}$ such that every $b \in \mathbb{Z}_{\ell}$ can be written as a sum of exactly $i$ distinct elements of $X$.

Construction. Let

$$
\ell \sim \frac{1}{6 i^{2} \ln k}\left(\begin{array}{l}
k \\
i
\end{array}\right) \geq \frac{1}{12 i^{2} \ln k}\left(\begin{array}{l}
k \\
i
\end{array}\right)
$$


be a prime number. Let $V$ be a set of $n$ vertices with an equipartition $V=V_{1} \cup V_{2} \cup \cdots \cup V_{\ell}$, and define $\sigma: V \rightarrow \mathbb{Z}_{\ell}$ by $\sigma(v)=j$ when $v \in V_{j}$. We associate an $X \subseteq V$ with the multi-set $\sigma(X) \subseteq \mathbb{Z}_{\ell}$ by $\sigma(X)=\{\sigma(v): v \in X\}$, and define the weight $w(X)=\Sigma_{v \in X} \sigma(v)(\bmod \ell)$. Let $\varphi$ be the weight with the fewest $k$-subsets having that weight. Finally, the edges of our hypergraph $G_{i}$ are

$$
\left\{X \in\left(\begin{array}{l}
V \\
k
\end{array}\right): w(X) \neq \varphi \text { and } \forall v \in X, \sigma(X-v) \text { is an } i \text {-fold sum cover of } \mathbb{Z}_{\ell}\right\} \text {. }
$$

The lower bounds in Theorems 2, 3, and 4 follow from the following two claims. Claim 6 implies that $G_{i}$ does not contain $B_{i+1}^{(k)}, B_{k-i}^{(k)}, \widehat{B}_{k-i}^{(k)}$, and in particular, $G_{1}$ does not contain $C^{(k)}$.

Claim 6. Let $F$ be a k-uniform hypergraph $(V, E)$ such that $V$ contains two disjoint subsets $S, T$ with $|T|=k-1$, and $|S|=i+1$ or $|S|=k-i$, and $E$ contains an edge containing $T$ and all $k$-sets $S \cup T^{\prime}$ with $T^{\prime} \in\left(\begin{array}{c}T \\ k-|S|\end{array}\right)$. Then $G_{i}$ is $F$-free.

Proof. We assume that $G$ contains a copy of $F$. Then $V(G)$ contains two disjoint sets $S$ and $T$ such that $|T|=k-1$ and $T$ is contained in an edge of $G$. By the definition of $G_{i}$, the set $\sigma(T)$ is an $i$-fold sum cover of $\mathbb{Z}_{\ell}$, namely, $\left\{w\left(T^{\prime}\right): T^{\prime} \in\left(\begin{array}{c}T \\ i\end{array}\right)\right\}$ covers all values of $\mathbb{Z}_{\ell}$.

By the definition of $F$ we have either $|S|=i+1$ or $|S|=k-i$. First assume that $|S|=i+1$. If we take all $k$-sets of the form $S \cup T^{\prime}$ where $T^{\prime} \subseteq T$ with $\left|T^{\prime}\right|=k-i-1$, then the set of weights of these $k$-sets will range over all elements of $\mathbb{Z}_{\ell}$ because $w\left(S \cup T^{\prime}\right)=w(S)+w(T)-w\left(T \backslash T^{\prime}\right)$, and $w\left(T \backslash T^{\prime}\right)$ can take any value of $\mathbb{Z}_{\ell}(w(S)$ and $w(T)$ are fixed). However, this is impossible since we explicitly prohibited one of the weights in the definition of $G_{i}$.

Now assume that $|S|=k-i$. If we take all of $k$-sets of the form $S \cup T^{\prime}$ where $T^{\prime} \subseteq T$ with $\left|T^{\prime}\right|=i$, then the set of weights of these $k$-sets will again range over all the elements of $\mathbb{Z}$ because $w\left(S \cup T^{\prime}\right)=w(S)+w\left(T^{\prime}\right)$ where $w\left(T^{\prime}\right)$ can take any value of $\mathbb{Z}_{\ell}(w(S)$ is fixed). However, this is impossible by the construction of $G_{i}$.

\section{Claim 7.}

$$
e\left(G_{i}\right) \geq\left(1-(1+o(1)) \frac{1}{\ell}\right)\left(\begin{array}{l}
n \\
k
\end{array}\right) \geq\left[1-\frac{12 i^{2} \ln k}{\left(\begin{array}{c}
k \\
i
\end{array}\right)}-o\left(\frac{\ln k}{k^{i}}\right)\right]\left(\begin{array}{l}
n \\
k
\end{array}\right) .
$$

Proof. By removing the edges with weight $\varphi$, we retain at least $1-\frac{1}{\ell}$ of the edges of $\left(\begin{array}{l}V \\ k\end{array}\right)$. On the other hand, by Lemma 8 below, with probability $1-o(1 / \ell)$ that an edge chosen uniformly at random from $\left(\begin{array}{l}V \\ k\end{array}\right)$ satisfies the property that all of its $k-1$ elements subsets are $i$-fold sum covers of $\mathbb{Z}_{\ell}$. It follows that the graph will have edge density at least $1-(1+o(1))(1 / \ell)$.

Lemma 8. Let $k \geq i \geq 1$ be integers and $\ell$ be a prime number satisfying (2). Let $y_{1}, y_{2}, \ldots, y_{k}$ be chosen uniformly and independently at random from $\mathbb{Z}_{\ell}$ (with replacement). Let $Y$ be the multiset $\left\{y_{1}, \ldots, y_{k}\right\}$. With probability $1-o(1 / \ell)$ every $(k-1)$-element subset of $Y$ is an $i$-fold sum cover of $\mathbb{Z}_{\ell}$.

Proof. We first consider the case when $i=1$. In this case $\ell \sim \frac{k}{3 \ln k}$ suffices. For $x \in \mathbb{Z}_{\ell}$ and $Y^{\prime} \in\left(\begin{array}{c}Y \\ k-1\end{array}\right)$, let $\mathcal{M}_{x, Y^{\prime}}$ be the event that $x \neq y$ for any $y \in Y^{\prime}$. We have

$$
\operatorname{Pr}\left(\mathcal{M}_{x, Y^{\prime}}\right)=\left(1-\frac{1}{\ell}\right)^{k-1} \leq e^{-(k-1) / \ell}=e^{-(3-o(1)) \ln k}=o\left(\ell^{-2} k^{-1}\right)
$$


Thus the probability that there exists a $(k-1)$-subset of $Y$ which is not a 1 -fold sum cover of $\mathbb{Z}_{\ell}$ is at most $\sum_{x, Y^{\prime}} \operatorname{Pr}\left(\mathcal{M}_{x, Y^{\prime}}\right)=o\left(\ell^{-2} k^{-1}\right) \cdot \ell k=o(1 / \ell)$.

We thus assume that $i \geq 2$ for the rest of our proof. We begin by studying the distribution of $(i-1)$-fold sums of elements of $Y$. For an arbitrary set $A \subseteq[k]$ we use the notation $\sum A$ for the sum $\sum_{a \in A} y_{a}$. For each $x \in \mathbb{Z}_{\ell}$ let $\mathcal{F}_{x}$ be the collection of sets $A \in\left({ }^{[k]}{ }_{i-1}\right)$ such that $\sum A=x$. Let $\mathcal{E}$ be the event that there exists an $x \in \mathbb{Z}_{\ell}$ such that $\left|\mathcal{F}_{x}\right| \geq 2 i+1$. We show that $\operatorname{Pr}(\mathcal{E})=o(1 / \ell)$. Note that this is a simple observation for $i=2$.

Let $i \geq 3$. For $x \in \mathbb{Z}_{\ell}$, define $\mathcal{E}_{x}$ to be the event that $\left|\mathcal{F}_{x}\right| \geq 2 i+1$. We further partition $\mathcal{E}_{x}$ into two cases.

Case 1: There exist $2 i+1(i-1)$-subsets $A_{1}, \ldots, A_{2 i+1}$ of $[k]$ such that $\sum A_{1}=\cdots=\sum A_{2 i+1}=x$ and $A_{1}, \ldots, A_{2 i+1}$ has a system of distinct representatives (SDR).

Case 2: There exist $2 i+1(i-1)$-subsets $A_{1}, \ldots, A_{2 i+1}$ of $[k]$ such that $\sum A_{1}=\cdots=\sum A_{2 i+1}=x$ and $A_{1}, \ldots, A_{2 i+1}$ do not have a SDR.

We bound the probability of each case by an application of the union bound. First, if $A_{1}, \ldots, A_{t}$ are distinct $(i-1)$-subsets of $[k]$ with a SDR, then

$$
\operatorname{Pr}\left(\sum A_{1}=\sum A_{2}=\cdots=\sum A_{t}=x\right)=\left(\frac{1}{\ell}\right)^{t}
$$

To see this, assume that $a_{1}, \ldots, a_{t}$ are representatives of $A_{1}, \ldots, A_{t}$. We rewrite

$$
\operatorname{Pr}\left(\sum A_{1}=\cdots=\sum A_{t}=x\right)=\prod_{j=1}^{t} \operatorname{Pr}\left(E_{j} \mid E_{1} \ldots E_{j-1}\right),
$$

where $E_{j}$ is the event that $\sum A_{j}=x$. Each term in this product equals to $1 / \ell$ because each $E_{j}$ sets the value of $y_{a_{j}}$. Using the fact $\left(\begin{array}{c}m \\ n\end{array}\right) \leq\left(\frac{e m}{n}\right)^{n}$ for all positive integers $m \geq n$ and (2), the probability of Case 1 is at most

$$
\left(\begin{array}{c}
\left(\begin{array}{c}
k \\
i-1
\end{array}\right) \\
2 i+1
\end{array}\right)\left(\frac{1}{\ell}\right)^{2 i+1} \leq\left(\frac{e\left(\begin{array}{c}
k \\
i-1
\end{array}\right)}{(2 i+1) \ell}\right)^{2 i+1} \leq\left(\frac{6 e i^{2} \ln k}{k-i+1}\right)^{2 i+1}=o\left(\frac{1}{\ell^{2}}\right)
$$

where the last equality uses the assumption $i \leq \ln k /(10 \ln \ln k)$.

Now consider Case 2. Assume that $A_{1}, \ldots, A_{2 i+1}$ are $(i-1)$-subsets of $[k]$ such that $\sum A_{1}=$ $\cdots=\sum A_{2 i+1}=x$ and $A_{1}, \ldots, A_{2 i+1}$ do not have a SDR. Let $B_{1}, \ldots, B_{b}, B_{b+1}$ be a minimal subfamily of $A_{1}, \ldots, A_{2 i+1}$ such that $\left|\cup_{j=1}^{b+1} B_{j}\right| \leq b$ (Hall's theorem guarantees the existence of such subfamily). Then $\left|\cup_{j=1}^{b} B_{j}\right| \leq\left|\cup_{j=1}^{b+1} B_{j}\right| \leq b$ and on the other hand, $\left|\cup_{j=1}^{b} B_{j}\right| \geq b$ by the minimality of $B_{1}, \ldots, B_{b+1}$. Thus $\left|\cup_{j=1}^{b} B_{j}\right|=b$. Since every proper subfamily of $B_{1}, \ldots, B_{b+1}$ satisfies Hall's condition, $B_{1}, \ldots, B_{b}$ has a SDR. Clearly $b \leq 2 i$. Furthermore, we claim that $b \geq i+1$. In fact, since $A_{1}, \ldots, A_{t}$ are $(i-1)$-sets, we have $b \geq\left|\cup_{\ell=1}^{b+1} B_{\ell}\right| \geq i-1 \geq 1$. Consequently $b \geq\left|B_{1} \cup B_{2}\right| \geq i$. Since an $i$-set has only $i$ distinct $(i-1)$-subsets, we have $b \geq\left|B_{1} \cup \cdots \cup B_{i+1}\right| \geq i+1$, as desired.

Therefore the probability of Case 2 is at most the probability that there exists $b(i-1)$-subsets $B_{1}, \ldots, B_{b}$ of $[k]$ for some $i+1 \leq b \leq 2 i$ such that

$$
\left|\cup_{j=1}^{b} B_{j}\right|=b, \sum B_{1}=\cdots=\sum B_{b}=x \text {, and } B_{1}, \ldots, B_{b} \text { have a SDR. }
$$


By (3), this probability is at most

$$
\sum_{b=i+1}^{2 i}\left(\begin{array}{c}
k \\
b
\end{array}\right)\left(\begin{array}{c}
\left(\begin{array}{c}
b \\
i-1
\end{array}\right) \\
b
\end{array}\right)\left(\frac{1}{\ell}\right)^{b} \leq \sum_{b=i+1}^{2 i}\left(\frac{e^{2} k\left(\begin{array}{c}
b \\
i-1
\end{array}\right)}{b^{2} \ell}\right)^{b} \leq i\left(\frac{e^{2} k\left(\begin{array}{c}
2 i \\
i-1
\end{array}\right)}{(i+1)^{2} \ell}\right)^{i+1}=o\left(\frac{1}{\ell^{2}}\right),
$$

where the last equality uses the assumption that $3 \leq i \leq \ln k /(10 \ln \ln k)$.

Adding the probabilities in the two cases together, we have $\operatorname{Pr}\left(\mathcal{E}_{x}\right)=o\left(\frac{1}{\ell^{2}}\right)$ and $\operatorname{Pr}(\mathcal{E}) \leq$ $\sum_{x \in \mathbb{Z}_{\ell}} \operatorname{Pr}\left(\mathcal{E}_{x}\right)=o\left(\frac{1}{\ell}\right)$.

Next, let us consider the $i$-fold sums of a fixed $(k-1)$-element subset of $Y$. We may assume that the elements of our $(k-1)$-element subset are $y_{1}, y_{2}, \ldots, y_{k-1}$. Let $S_{j}$ be the set of elements of $\mathbb{Z}_{\ell}$ covered by $(i-1)$-fold sums of $\left\{y_{1}, \ldots, y_{j}\right\}$. Note that if the event $\mathcal{E}$ does not hold then $\left|S_{j}\right| \geq \frac{1}{2 i}\left(\begin{array}{c}j \\ i-1\end{array}\right)$. For $x \in \mathbb{Z}_{\ell}$ let $\mathcal{M}_{x}$ be the event that $x$ does not appear as an $i$-fold sum of $y_{1}, \ldots, y_{k-1}$. We have

$$
\begin{aligned}
\operatorname{Pr}\left(\mathcal{M}_{x} \cap \overline{\mathcal{E}}\right) & =\prod_{j=i}^{k-1} \operatorname{Pr}\left(\forall A \in\left(\begin{array}{c}
{[j-1]} \\
i-1
\end{array}\right), y_{j}+\sum A \neq x\right)=\prod_{j=i}^{k-1}\left(1-\frac{\left|S_{j-1}\right|}{\ell}\right) \\
& \leq \exp \left\{-\sum_{j=i}^{k-1} \frac{1}{2 i}\left(\begin{array}{c}
j-1 \\
i-1
\end{array}\right) \frac{1}{\ell}\right\}=\exp \left\{-\frac{1}{2 i} \cdot \frac{1}{\ell} \cdot\left(\begin{array}{c}
k-1 \\
i
\end{array}\right)\right\} \\
& =\exp \{-(3-o(1)) i \ln k\} \leq o\left(\ell^{-2} k^{-1}\right) .
\end{aligned}
$$

Summing over all $x \in \mathbb{Z}_{\ell}$ and all $(k-1)$-element subsets of $Y$ gives the desired result.

\section{Upper bounds}

Proof of the Upper Bound in Theorem 2. We shall apply a result of Sidorenko [11] that for a $k$ uniform hypergraph $F$ with $f$ edges,

$$
\pi(F) \leq 1-\frac{1}{f-1}
$$

Therefore, for $2 \leq i \leq k$,

$$
\pi\left(B_{i}^{(k)}\right) \leq 1-\frac{1}{i+\left(\begin{array}{c}
k-1 \\
i-1
\end{array}\right)-1} .
$$

Thus for fixed $i$ and $k \rightarrow \infty$, we have

$$
\pi\left(B_{i+1}^{(k)}\right) \leq 1-\frac{1}{i+\left(\begin{array}{c}
k-1 \\
i
\end{array}\right)}, \quad \text { and } \quad \pi\left(B_{k-i}^{(k)}\right) \leq 1-\frac{1}{k-i+\left(\begin{array}{c}
k-1 \\
i
\end{array}\right)-1} .
$$

In order to prove the upper bound of Theorem 3, shall count the number of complete subhypergraphs of a dense hypergraph. Fix a $k$-uniform hypergraph $G$ of order $n$. Let $m_{i}$ denote the number of $i$-cliques $K_{i}^{k}$ for $i \geq k$ in $G$, for example $m_{k}$ is the number of hyperedges of $G$. In addition, define $m_{k-1}=\left(\begin{array}{c}n \\ k-1\end{array}\right)$. Adapting the techniques previously used by Moon and Moser, de Caen [2] provided the following recursive lower bound on $m_{i}$. 
Theorem 9. If $m_{i-1}>0$, then

$$
m_{i+1} \geq \frac{i^{2} m_{i}}{(i-k+1)(i+1)}\left(\frac{m_{i}}{m_{i-1}}-\frac{(k-1)(n-i)+i}{i^{2}}\right) .
$$

This implies the following lower bound on $m_{\ell}$ for $\ell>k$.

Corollary 10. Let $0 \leq \alpha \leq 1$, and let $G$ be a k-uniform hypergraph with at least $(1-\alpha)\left(\begin{array}{l}n \\ k\end{array}\right) \frac{n}{n-k+1}$ edges. Then for any $\ell \geq k$,

$$
m_{\ell} \geq\left(\begin{array}{l}
n \\
\ell
\end{array}\right) \prod_{i=k}^{\ell}\left(1-\left(\begin{array}{l}
i-1 \\
k-1
\end{array}\right) \alpha\right)
$$

Proof. The $k=2$ case can be found in Lovász [9] (Problem 10.40). The proof of the general case is almost the same. Let $a_{i}=m_{i} / m_{i-1}$. We claim that for $i \geq k$,

$$
a_{i} \geq \frac{n}{i}\left(1-\left(\begin{array}{c}
i-1 \\
k-1
\end{array}\right) \alpha\right) .
$$

Let us prove (4) by induction. By the definition of $m_{k}$ and $m_{k-1}$, we have

$$
a_{k} \geq(1-\alpha)\left(\begin{array}{l}
n \\
k
\end{array}\right) \frac{n}{n-k+1} \frac{1}{\left(\begin{array}{c}
n \\
k-1
\end{array}\right)}=(1-\alpha) \frac{n}{k}
$$

For $i>k$, by Theorem 9 and the induction hypothesis,

$$
\begin{aligned}
a_{i+1} & \geq \frac{i^{2}}{(i-k+1)(i+1)}\left(a_{i}-\frac{(k-1)(n-i)+i}{i^{2}}\right) \\
& \geq \frac{i^{2}}{(i-k+1)(i+1)}\left(\frac{n}{i}\left(1-\left(\begin{array}{c}
i-1 \\
k-1
\end{array}\right) \alpha\right)-\frac{n}{i^{2}}(k-1)\right) \\
& =\frac{n}{(i-k+1)(i+1)}\left(i\left(1-\left(\begin{array}{c}
i-1 \\
k-1
\end{array}\right) \alpha\right)-k+1\right) \\
& =\frac{n}{i+1}\left(1-\left(\begin{array}{c}
i \\
k-1
\end{array}\right) \alpha\right) .
\end{aligned}
$$

Let $\ell \geq k$. By applying (4) to $i=k, \ldots, \ell$, we obtain

$$
m_{\ell}=m_{k-1} \prod_{i=k}^{\ell} a_{i} \geq\left(\begin{array}{c}
n \\
k-1
\end{array}\right) \prod_{i=k}^{\ell} \frac{n}{i}\left(1-\left(\begin{array}{c}
i-1 \\
k-1
\end{array}\right) \alpha\right) \geq\left(\begin{array}{c}
n \\
\ell
\end{array}\right) \prod_{i=k}^{\ell}\left(1-\left(\begin{array}{c}
i-1 \\
k-1
\end{array}\right) \alpha\right) .
$$

Proof of the Upper Bound in Theorem 3. Suppose that $G$ is a $\widehat{B}_{k-i}^{(k)}$-free $k$-uniform hypergraph on $n$ vertices with at least $(1-\alpha) \frac{n}{n-k+1}\left(\begin{array}{l}n \\ k\end{array}\right)$ edges, where

$$
\alpha=\frac{\ln \left[2\left(\begin{array}{c}
k-1 \\
i
\end{array}\right)\right]-\ln \ln \left[2\left(\begin{array}{c}
k-1 \\
i
\end{array}\right)\right]}{2\left(\begin{array}{c}
k-1 \\
i
\end{array}\right)} \approx \frac{i !}{2 k^{i}}(i \ln k-\ln \ln k) .
$$


By standard averaging arguments, there exists a subset $A$ of size $k-i$ so that the neighborhood of $A$ (i.e., the collection of $i$-sets whose union with $A$ is an edge of $G$ ) is an $i$-uniform hypergraph on $n-(k-i)$ vertices with at least $(1-\alpha) \frac{n}{n-k+1}\left(\begin{array}{c}n-(k-i) \\ i\end{array}\right)>(1-\alpha) \frac{n-(k-i)}{n-k+1}\left(\begin{array}{c}n-(k-i) \\ i\end{array}\right)$ edges. From Corollary 10 it follows that the neighborhood of $A$ has at least $f(\alpha)\left(\begin{array}{c}n-(k-i) \\ k-1\end{array}\right)(k-1)$-cliques, where

$$
f(\alpha):=\prod_{j=i}^{k-1}\left(1-\left(\begin{array}{l}
j-1 \\
i-1
\end{array}\right) \alpha\right) .
$$

No such clique can be extended to an edge of $G$ by adding a vertex outside $A$ - otherwise we obtain a copy of $\widehat{B}_{k-i}^{(k)}$. The number of non-edges in $G$ is at most

$$
\left(\alpha-\frac{k-1}{n-k+1}(1-\alpha)\right)\left(\begin{array}{l}
n \\
k
\end{array}\right) \geq f(\alpha)\left(\begin{array}{c}
n-(k-i) \\
k-1
\end{array}\right) \frac{n-j-(k-1)}{k},
$$

where the term $n-j-(k-1)$ counts the number of remaining vertex outside $A$ and a $(k-1)$-clique and thus each non-edge may be counted at most $k$ times. As $n \rightarrow \infty$, (6) becomes

$$
(\alpha+o(1))\left(\begin{array}{l}
n \\
k
\end{array}\right) \geq(f(\alpha)+o(1))\left(\begin{array}{l}
n \\
k
\end{array}\right)
$$

which implies that $\alpha \geq f(\alpha)$. It is easy to see that $1-x \geq e^{-2 x}$ for $0 \leq x \leq \frac{\ln 2}{2}$. By (5), we have $\left(\begin{array}{c}k-2 \\ i-1\end{array}\right) \alpha \rightarrow 0$ as $k \rightarrow \infty$, and so

$$
f(\alpha) \geq \prod_{j=i}^{k-1} \exp \left(-2\left(\begin{array}{c}
j-1 \\
i-1
\end{array}\right) \alpha\right)=\exp \left(-2\left(\begin{array}{c}
k-1 \\
i
\end{array}\right) \alpha\right)
$$

Consequently,

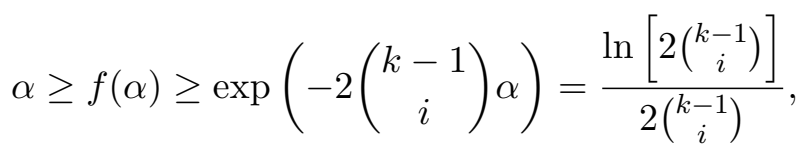

contradicting (5).

Proof of the Upper Bound in Theorem 4. A forest is a hypergraph whose edges can be ordered as $E_{1}, E_{2}, \ldots, E_{m}$ such that for each $1 \leq i \leq m$, we have $E_{i} \cap\left(E_{1} \cup \cdots \cup E_{i-1}\right) \subseteq E_{j}$ for some $j<i$. Based on the work of Sidorenko [11], Keevash [5] showed that if a $k$-uniform hypergraph $H$ has $f$ edges and the maximal number of edges of $H$ forming a forest is $t$ then its Turán density $\pi=\pi(H)$ satisfies

$$
\pi^{t-1}-(f-t)(1-\pi) \leq 0 .
$$

Notice that $C^{(k)}$ has $k$ edges, $k-1$ of which form a forest, so $t=k-1$ and $f-t=1$. Hence the Turán density of $C^{(k)}$ satisfies

$$
\pi^{k-2}-(1-\pi) \leq 0
$$

Letting $\pi=1-\alpha$ this becomes

$$
(1-\alpha)^{k-2} \leq \alpha .
$$


As seen in the proof of the upper bound in Theorem 3, this implies $\alpha \geq e^{-2(k-2) \alpha}$, which further implies that

$$
\alpha \geq \frac{\ln [2(k-2)]-\ln \ln [2(k-2)]}{2(k-2)} .
$$

Hence $\pi=1-\alpha \leq 1-\frac{\ln k}{2 k}+O\left(\frac{\ln \ln k}{2 k}\right)$, as desired.

Acknowledgments: This paper grew out of a problem group at the AIM Hypergraph Turán problem workshop held in March 2011. We thank AIM, the NSF, and the organizers Dhruv Mubayi, Oleg Pikhurko, and Benny Sudakov for organizing the workshop. We especially thank Dhruv Mubayi for the suggestion of the problem which led to this note. We also thank for Emily Allen, Steve Butler, Éva Czabarka, Huang Hao and Penny Haxell who participated in the discussions during the workshop.

\section{References}

[1] Tom Bohman, Alan Frieze, Dhruv Mubayi and Oleg Pikhurko, Hypergraphs with independent neighborhoods, Combinatorica 30 (2010), 277-293.

[2] Dominique de Caen, Extension of a theorem of Moon and Moser on complete subgraphs. Ars Combin. 16 (1983), 5-10.

[3] Paul Erdős, On the combinatorial problems which I would like to see solved, Combinatorica 1 (1981), 25-42.

[4] Victor Falgas-Ravry and Emil R. Vaughan, On applications of Razborov's flag algebra calculus to extremal 3-graph theory, arXiv:1110.1623v1.

[5] Peter Keevash, The Turán problem for hypergraphs on fixed size, Electronic Journal of Combinatorics 12 (2005), Note 11, 6pp. (electronic)

[6] Ki Hang Kim and Fred W. Roush, On a problem of Turán, in: Studies in pure mathematics, Birkhäuser, Boston, 1983, 423-425.

[7] Alexandr V. Kostochka, A class of constructions for Turán's (3,4)-problem, Combinatorica 2 (1982), 187-192.

[8] Linyuan Lu and Yi Zhao, An exact result and its application on hypergraph Turán numbers, SIAM Journal on Discrete Mathematics 23 (2009), 1324-1334.

[9] László Lovász, Combinatorial problems and exercises. Second edition. North-Holland Publishing Co., Amsterdam, 1993.

[10] Alexander Razborov, On 3-hypergraphs with forbidden 4-vertex configurations, SIAM Journal on Discrete Mathematics 24 (2010), 946-963.

[11] Alexander Sidorenko, An analytic approach to extremal problems for graphs and hypergraphs, in: Extremal problems for finite sets (Visegrád 1991), Bolyai Soc. Math. Stud., 3, János Bolyai Math. Soc., Budapest, 423-455. 
[12] Alexander Sidorenko, Upper bounds for Turán numbers, J. Combin. Theory Ser. A 77 (1997), 134-147.

[13] Pál Turán, On an extremal problem in graph theory. Mat. Fiz. Lapok 48 (1941), 436-452. 